Article

\title{
Incorporation of Rutin in Electrospun Pullulan/PVA Nanofibers for Novel UV-Resistant Properties
}

\author{
Yongfang Qian ${ }^{1,2}$, Mengjie Qi ${ }^{1}$, Laijiu Zheng ${ }^{1}$, Martin W. King ${ }^{2}$, Lihua Lv ${ }^{1}$ and Fang Ye ${ }^{1, *}$ \\ 1 School of Textile and Material Engineering, Dalian Polytechnic University, Dalian 116034, China; \\ qianyf@dlpu.edu.cn (Y.Q.); qimengjie123@gmail.com (M.Q.); fztrxw@dlpu.edu.cn (L.Z.); \\ lvlh@dlpu.edu.cn (L.L.) \\ 2 College of Textiles, North Carolina State University, Raleigh, NC 27695, USA; martin_king@ncsu.edu \\ * Correspondence: yidi999@163.com; Tel: +86-139-4095-6798
}

Academic Editor: Nicole Zander

Received: 29 April 2016; Accepted: 14 June 2016; Published: 23 June 2016

\begin{abstract}
This study aimed to investigate the incorporation of rutin into electrospun pullulan and poly(vinyl alcohol) (PVA) nanofibers to obtain ultraviolet (UV)-resistant properties. The effect of weight ratios between pullulan and PVA, and the addition of rutin on the nanofibers' morphology and diameters were studied and characterized by scanning electron microscopy (SEM). Fourier transform infrared (FTIR) analysis was utilized to investigate the interaction between pullulan and PVA, as well as with rutin. The results showed that the inclusion of PVA results in the increase in the fiber's diameter. The addition of rutin had no obvious effect on the fibers' average diameters when the content of rutin was less than $7.41 \%$. FTIR results indicated that a hydrogen bond formed between pullulan and PVA, also between these polymers and rutin. Moreover, the addition of rutin could enhance the mechanical properties due to its stiff structure and could decrease the transmittance of UVA and UVB to be fewer than 5\%; meanwhile, the value of ultraviolet protection factor (UPF) reached more than 40 and 50 when the content of rutin was $4.46 \%$ and $5.67 \%$, respectively. Therefore, the electrospun pullulan/PVA/rutin nanofibrous mats showed excellent UV resistance and have potential applications in anti-ultraviolet packaging and dressing materials.
\end{abstract}

Keywords: pullulan; rutin; electrospinning; nanofiber; UV resistance

\section{Introduction}

Electrospinning is a simple and effective method to fabricate nonwoven mats with large surface areas and porosities [1,2]. In this method, high voltage is applied on the drop to the tip of the needle as the draft force on the polymer solution or melt. When the applied electrical force overcomes the critical surface tension of the polymer liquid, the liquid is ejected from the nozzle, stretched, and finally deposited on the grounded collector in the form of nonwoven mats with fibers ranging from tens of microns to nanometers in diameter [3]. The effected parameters on the fiber formation and diameters in the electrospinning process involve characteristics of the solution (e.g., electric conductivity, viscosity, surface tension, and concentration), controlled variables (e.g., tip-to-collector distance, voltages, and feeding rate), and the atmosphere (mainly the humidity and the temperature) [4].

Pullulan is an extracellular microbial polysaccharide produced by the yeast-like fungus Aureobasidium pullulans [5,6]. The basic chemical structure is a linear maltotriose unit connected by $\alpha-1,6$ linkages, while the internal glucose unit within maltotriose is a $\alpha-1,4$ glycosidic linkage. Remarkably, this structure gives pullulan excellent solubility and high resilience in terms of structure [7]. Islam et al. [8] and Karim et al. [9] investigated the incorporation of PVA and montmorillonite into pullulan nanofibers to improve the mechanical properties and thermal performance of electrospun 
nanofibrous mats. Islam et al. [10] and Karim et al. [11] studied functionalized pullulan with fluorinated silane to fabricate super hydrophobic membranes.

Poly(vinyl alcohol) (PVA) is a highly biocompatible and nontoxic polymer with good water-solubility, which is influenced by the alcoholysis degree and degree of polymerization. The unique properties lead to the use of PVA in a wide range of applications such as the food, packaging, cosmetic, spinning, and paper-making industries [12]. PVA is usually used as the strong part to improve or modify the physicochemical properties in electrospinning. Mohmoodi et al. [13] found that the addition of PVA to the solution of chitosan can improve the spinnability; moreover, the resultant nanofibrous mats have good absorbent ability and can be applied in the removal of dye from colored wastewater. Sousa et al. [14] introduced PVA to the agar solution, and Wang et al. [15,16] incorporated PVA into honey and milk to improve the spinnability. In addition, Wang et al. [17] demonstrated that incorporation of pleurocidin into electrospun PVA nanofibers can preserve the bioactivity of pleurocidin and realize sustained release to improve food safety.

Rutin, $3^{\prime}, 4^{\prime}, 5,7$-tetrahydroxyflavone-3 $\beta$-D-rutinoside, is one of the most abundant natural flavonoids and has many biological properties, being anti-inflammatory, antiallergenic, and antimicrobial $[18,19]$. Rutin was found to be photo-stable and capable of enhancing the defense system against environment stresses, including low temperature, UV light, and desiccation [20,21]. Xing et al. [22] proposed that rutin be added to poly(L-lactide-co-glycolide) (PLGA) solution and then electrospun to obtain antibacterial nanofibrous mats. However, reports related to the UV resistance of rutin-contained nanofibrous mats are scarce. This study aimed to investigate the incorporation of rutin into the pullulan- and PVA-blended nanofibers. The morphologies of electrospun pullulan and PVA nanofibers, and the effect of introducing rutin onto the formed fibers, were observed via scanning electron microscopy (SEM). Fourier transform infrared (FTIR) spectrophotometer was utilized to analyze the interaction between pullulan and PVA, as well as the interaction between these polymers and rutin. Finally, the ultraviolet (UV)-resistant properties were tested with a UV performance tester.

\section{Results and Discussion}

\subsection{Morphology of Electrospun Pullulan and PVA Nanofibers}

The concentration or the corresponding viscosity was one of the most effective variables for controlling the fiber morphology [23]. A higher concentration results in higher viscosity. Fibers with different weight ratios of pullulan and PVA (Figure 1) at the same total concentration of $0.15 \mathrm{~g} / \mathrm{mL}$ show smooth and uniform morphology. When the contents of the PVA increased from $0 \%, 25 \%$, $50 \%$, to $75 \%$, the average diameters were $89,171,311$ and $413 \mathrm{~nm}$, respectively. The diameters of the fibers increased due to the increased viscosity. Pure PVA failed to be electrospun at a concentration of $0.15 \mathrm{~g} / \mathrm{mL}$ since the solution was too viscous to be drafted. Therefore, the incorporation of PVA was capable of improving the spinnability of pullulan.
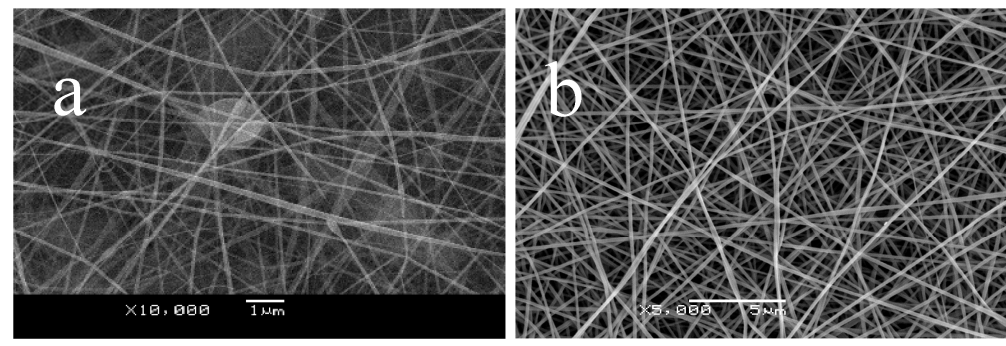

Figure 1. Cont. 


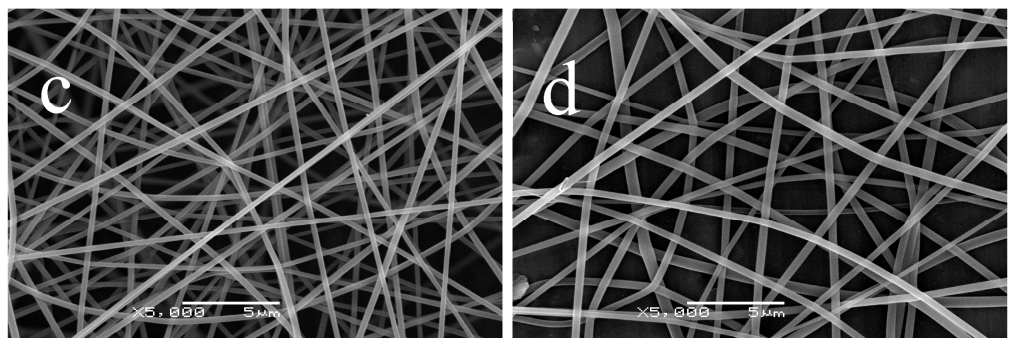

Figure 1. Scanning electron microscopy (SEM) micrographs of electrospun pullulan/poly(vinyl alcohol) (PVA) blended fibrous mats at a concentration of $0.15 \mathrm{~g} / \mathrm{mL}$ with weight ratios of (a) 100/0; (b) 75/25; (c) $50 / 50 ;$ (d) $25 / 75$.

\subsection{The Effect of Rutin on Electrospun Pullulan and PVA Nanofibers}

The average diameter of an electrospun pullulan and PVA nanofibrous mat with a weight ratio of $50 / 50$ was $311 \mathrm{~nm}$. When rutin was added to the spinning solution and then electrospun, the collected nanofibrous mats became light yellow compared with the original white mats. The morphologies of electrospun pullulan and PVA nanofibers containing different amounts of rutin are shown in Figure 2. When the contents of rutin were $3.23 \%, 4.46 \%, 5.67 \%$, and $7.41 \%(w / w)$ compared to pullulan and PVA blends, the average diameters were 312, 322, 326 and $334 \mathrm{~nm}$, respectively, which indicated that the incorporation and the amount of rutin had no obvious influence on the morphology or the diameters of the electrospun nanofibers. However, when the content of rutin reached $8.54 \%(w / w)$, the morphology exhibited spindle-like beads between the fibers, which means that the spinnability had been affected. It is noted that the rutin cannot be fabricated into any type of fiber without other polymer materials. Therefore, there would be no fibers collected on the grounded plate if the weight ratio of rutin exceeded a limited value.
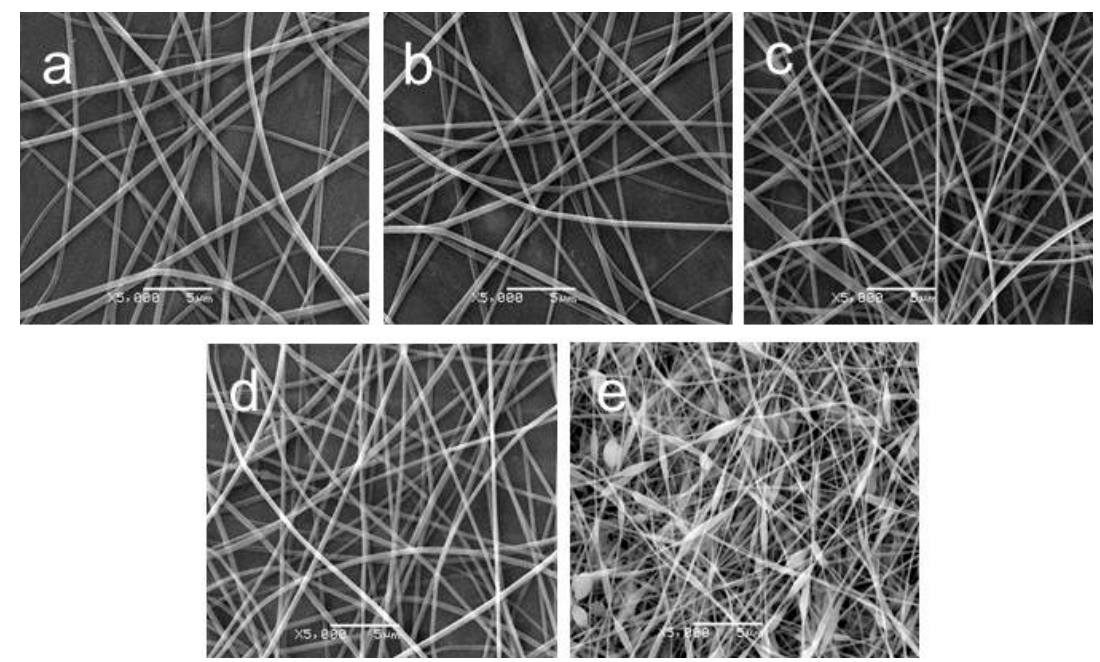

Figure 2. SEM micrographs of electrospun pullulan/PVA nanofibers containing different amounts of rutin (w/w): (a) $3.23 \%$; (b) $4.46 \%$; (c) $5.67 \%$; (d) $7.41 \%$; (e) $8.54 \%$.

\subsection{FTIR Analysis of Electrospun Pullulan/PVA and Pullulan/PVA/Rutin Nanofibers}

The FTIR spectra gave information about information about the structure and interaction of the blended membranes studied. The spectra of pure pullulan, PVA, and pullulan-/PVA-blended membranes in the range of $4000-600 \mathrm{~cm}^{-1}$ (Figure 3a) and 2000-600 $\mathrm{cm}^{-1}$ (Figure $3 \mathrm{~b}$ ) are shown. Pure PVA membrane exhibits an identical strong absorption peak located at $1089 \mathrm{~cm}^{-1}$, indicating the presence of $\mathrm{C}-\mathrm{O}$. The absorption peak at $2939 \mathrm{~cm}^{-1}$ is due to the stretching vibration of the $\mathrm{CH}_{2}$ group. 
The broad absorption peak at $3330 \mathrm{~cm}^{-1}$ is attributed to the stretching vibration of hydroxyl group $(-\mathrm{OH})$. The spectrum of pure pullulan has a strong absorption band at $848 \mathrm{~cm}^{-1}$, which is characteristic of the $\alpha$-glucopiranosid units. The band at $754 \mathrm{~cm}^{-1}$ proves the presence of $\alpha-(1,4)$ glucosidic bonds, and the band at $930 \mathrm{~cm}^{-1}$ demonstrates the presence of $\alpha-(1,6)$ glucosidic bands $[8,9]$. The band at $2930 \mathrm{~cm}^{-1}$ is caused by the stretching vibrations of methyl or methylene groups. Bands attributed to $\mathrm{CH} / \mathrm{CH}_{2}$ deformation vibrations occur in the range of $1300-1500 \mathrm{~cm}^{-1}$. The broad adsorption peak at $3330 \mathrm{~cm}^{-1}$ is also assigned to the hydroxyl group $(-\mathrm{OH})$. Comparing the spectra of pure pullulan and PVA, the absorption peak of the hydroxyl group $(-\mathrm{OH})$ in the spectra of the electrospun pullulan/PVA blend shifts from 3330 to $3320 \mathrm{~cm}^{-1}$, which is evidence that a hydrogen bond formed between the pullulan and PVA molecules. Therefore, the FTIR spectroscopy is an effective way of examining the interaction between polymers.
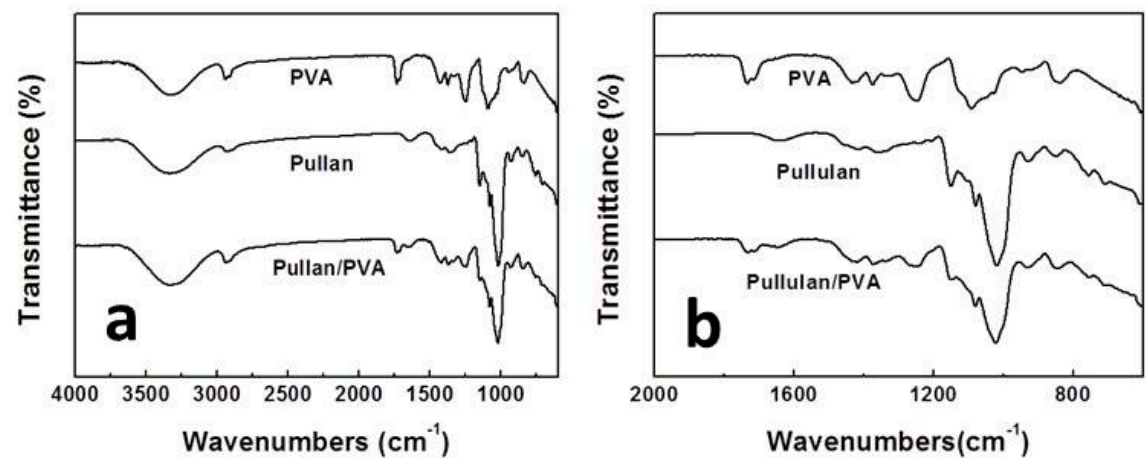

Figure 3. Fourier transform infrared (FTIR) spectra of PVA, pullulan and blended nanofibrous mats in wavenumbers range of (a) $4000-600 \mathrm{~cm}^{-1}$ and (b) $2000-600 \mathrm{~cm}^{-1}$.

Figure 4 shows the spectra of rutin, the electrospun pullulan/PVA, and the pullulan/PVA/rutin membranes. The spectrum of the rutin has absorption peaks at 3330 and $1359 \mathrm{~cm}^{-1}$, corresponding to the $\mathrm{O}-\mathrm{H}$ stretching vibrations of intercalated water. The adsorption peak located at $1650 \mathrm{~cm}^{-1}$ is due to the carbonyl stretching vibration. The peaks located at 1203,1123 and $1089 \mathrm{~cm}^{-1}$ represent a C-O-C bond stretching in the ethyl dioxy ring deformation. The peaks at 1504 and $1359 \mathrm{~cm}^{-1}$ are attributed to the asymmetric stretching of $\mathrm{C}=\mathrm{C}$ bond and the inter-ring stretching of $\mathrm{C}-\mathrm{C}$. The peak located at $1057 \mathrm{~cm}^{-1}$ is due to the epoxide group stretching. When rutin was added into the pullulan/PVA nanofibers, the FTIR spectra of the electrospun pullulan/PVA/rutin membrane exhibited a slight change compared with that of pullulan/PVA. The absorption band of the hydroxyl group became stronger and shifted from 3320 to $3306 \mathrm{~cm}^{-1}$, which also indicated the formation of hydrogen between rutin and the pullulan/PVA molecules.

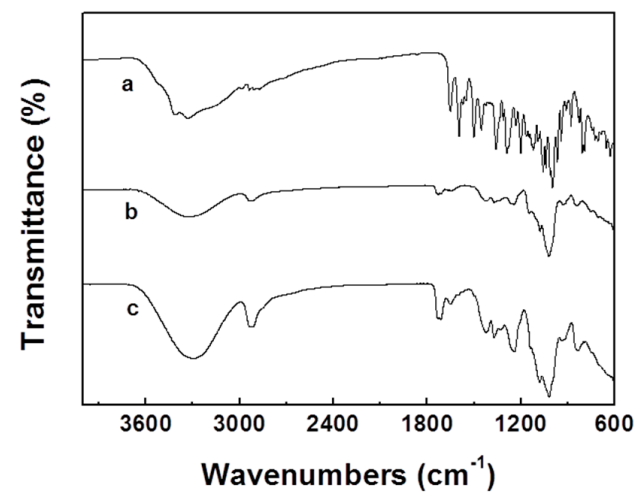

Figure 4. FTIR spectra of the rutin (a), electrospun pullulan/PVA (b) and pullulan/PVA/rutin (c) nanofibrous membranes. 


\subsection{Mechanical Properties of PVA/Pullulan and PVA/Pullulan/Rutin Nanofibrous Mats}

The stress and strain values of electrospun pullulan and PVA nanofibrous mats at different weight ratios are summarized in Table 1 . When the weight percentage of PVA was $0 \%, 25 \%, 50 \%$, and $75 \%$, the average ultimate tensile stress was $1.07,1.72,1.94$, and $2.56 \mathrm{MPa}$, while the average strain was $31 \%, 28 \%, 25 \%$, and $19 \%$, respectively. The average stress increased as the content of PVA increased, but the average strain decreased. Synthetic polymers usually have better spinnability and mechanical properties; thus, the incorporation of synthetic polymers with natural materials is an effective way of enhancing the formation of fibers and mechanical properties [24,25].

Table 1. The tensile properties of electrospun pullulan and PVA nanofibrous mats with different weight ratios $(n=3)$.

\begin{tabular}{ccccc}
\hline Pullulan/PVA & $\mathbf{1 0 0 / 0}$ & $\mathbf{7 5 / 2 5}$ & $\mathbf{5 0 / 5 0}$ & $\mathbf{2 5 / 7 5}$ \\
\hline Stress $(\mathrm{MPa})$ & $1.07 \pm 0.062$ & $1.72 \pm 0.125$ & $1.94 \pm 0.09$ & $2.56 \pm 0.147$ \\
Strain $(\%)$ & $31 \pm 3.606$ & $28 \pm 4.583$ & $25 \pm 5.568$ & $19 \pm 2.646$ \\
\hline
\end{tabular}

The effect of incorporating rutin into the mechanical properties of electrospun pullulan/PVA nanofibrous mats are summarized in Table 2 . When the weight percentage of rutin was $0 \%, 3.23 \%$, $4.46 \%, 5.67 \%$, and $7.41 \%$, the average ultimate tensile stresses were $1.94,2.38,2.85,3.22$, and $3.1 \mathrm{MPa}$, and the average stains were $25 \%, 23 \%, 21 \%, 16 \%$, and $12 \%$, respectively. It is noted that the electrospun pullulan/PVA/rutin nanofibrous mats had greater tensile stress than that of pullulan/PVA, and the ultimate tensile strength exhibited an increasing trend as the content of rutin increased, which indicated that the addition of rutin was capable of enhancing the mechanical properties of the nanofibrous mats. Rutin has stiff aromatic rings and ethylene dioxy rings in its structure [26]; meanwhile, rutin was capable of randomly dispersing in the electrospun nanofibers [22]. Thus, the addition of a limited amount of rutin can enhance the strength to some extent. Combined with the SEM morphology, the incorporation of rutin into the nanofibers can enhance the mechanical properties but has no obvious influence on the formation and diameter of fibers.

Table 2. The tensile properties of electrospun nanofibrous mats with different weight percentages of rutin compared to pullulan/PVA $(n=3)$.

\begin{tabular}{cccccc}
\hline Rutin (\%) & $\mathbf{0}$ & $\mathbf{3 . 2 3}$ & $\mathbf{4 . 4 6}$ & $\mathbf{5 . 6 7}$ & $\mathbf{7 . 4 1}$ \\
\hline Stress (MPa) & $1.94 \pm 0.09$ & $2.38 \pm 0.274$ & $2.85 \pm 0.298$ & $3.22 \pm 0.368$ & $3.1 \pm 0.171$ \\
Strain (\%) & $25 \pm 5.568$ & $23 \pm 2$ & $21 \pm 2.646$ & $16 \pm 2.646$ & $12 \pm 3$ \\
\hline
\end{tabular}

\subsection{The Ultraviolet-Resistant Property of the Electrospun Nanofibrous Mats Containing Rutin}

UV radiation consists of ultraviolet A (UVA), ultraviolet B (UVB), and ultraviolet C (UVC), all of which represent the regions 315-400 nm, 280-315 nm, and 200-280 nm, respectively. Excessive exposure to solar UVA and UVB radiation can cause skin cancers [20]. UVA and UVB transmittances, as well as ultraviolet protection factor (UPF), were tested with a spectrophotometer, and the results are summarized in Table 3. For pure rutin, the UVA and UVB transmittances, as well as the UPF, were $0.34 \pm 0.17,0$, and more than 50 , respectively. Generally, the transmittances of UVA and UVB decreased while the UPF markedly increased and the content of rutin increased. When the addition of rutin was greater than $3.23 \%$, the transmittances of UVA and UVB of electrospun pullulan/PVA/rutin nanofibrous mats were lower than $5 \%$. The UPF was above 40 when the content of rutin was $4.46 \%$ and was more than 50 when the content was $5.67 \%$. The UV-blocking effect was superior to traditional fabrics treated with $\mathrm{ZnO}$ or $\mathrm{TiO}_{2}$, of which the transmittance was less than $20 \%$ and the UPF was above 50 [27]. Both the UVA and UPF met the requirements and standards of UV-resistant products. 
The UV resistance of the electrospun nanofibrous mats is applicable to anti-ultraviolet packaging and dressing materials.

Table 3. The UVA, UVB transmittance and UPF of electrospun pullulan/PVA nanofibrous mats with different contents of rutin $(n=3)$.

\begin{tabular}{ccccccc}
\hline Rutin (\%) & $\mathbf{0}$ & $\mathbf{3 . 2 3}$ & $\mathbf{4 . 4 6}$ & $\mathbf{5 . 6 7}$ & $\mathbf{7 . 4 1}$ & $\mathbf{8 . 5 4}$ \\
\hline $\mathrm{T}_{\text {(UVA) }}(\%)$ & $6.06 \pm 0.68$ & $3.34 \pm 0.36$ & $2.44 \pm 0.8$ & $2.24 \pm 0.07$ & $0.76 \pm 0.15$ & $0.53 \pm 0.17$ \\
$\mathrm{~T}_{\text {(UVB) }}(\%)$ & $5.29 \pm 0.69$ & $2.75 \pm 0.36$ & $1.8 \pm 0.82$ & $2.25 \pm 0.07$ & $0.9 \pm 0.10$ & $0.61 \pm 0.23$ \\
UPF & $23 \pm 2.75$ & $29 \pm 2.45$ & $>40$ & $>50$ & $>50$ & $>50$ \\
\hline
\end{tabular}

\section{Materials and Methods}

\subsection{Materials}

Pullulan, food grade, was purchased from Hayashibara Biochemical Laboratories Inc. (Okayama, Japan). PVA in analytical pure grade was obtained from Kelong Chemical Reagent Factory (Chengdu, China). Rutin was purchased from Aladdin Industrial Corporation (Shanghai, China) with a purity of $98 \%$. Distilled water was used as the solvent to prepare all solutions.

\subsection{Preparation of the Spinning Solution and Electrospinning}

Pullulan and PVA powder were dissolved together in distilled water with different weight ratios of $100 / 0,75 / 25,50 / 50,25 / 75$, and $0 / 100$, separately, at total concentrations of $0.15 \mathrm{~g} / \mathrm{mL}$. Rutin was dissolved in a pullulan-/PVA-blended solution (50/50) with a weight percentage at 3.23\%, $4.46 \%$, $5.67 \%, 7.41 \%$, and $8.54 \%$, respectively, compared to the pullulan and PVA blends. All the solutions were stirred for $24 \mathrm{~h}$ at a temperature of $30^{\circ} \mathrm{C}$.

The blended polymer solutions were electrospun using a high voltage of $24 \mathrm{kV}$ supplied by a high voltage power supply (JDF-1, Beijing, China) purchased from the BMEI Co., Ltd. The feeding rate of the solution was set at $0.6 \mathrm{~mL} / \mathrm{h}$ and controlled by an accurate syringe driver (789100C, Cole-Parmer, Vernon Hills, IL, USA). The membranes of the nanofibers were collected with aluminum platinum paper, which was faced vertically to the needle tip with a tip-to-collector distance of $12 \mathrm{~cm}$. The electrospinning process was conducted at a room temperature of $30^{\circ} \mathrm{C}$ and a relative humidity of $45 \%$.

\subsection{Characterization of the Membranes}

The morphologies of electrospun nanofibers were observed via scanning electron microscopy (SEM, JSM 6040, JEOL, Tokyo, Japan) after being sprayed with platinum. The diameters were conducted by image visualization software Image J (National Institutes of Health, Bethesda, MD, USA). A Fourier transform infrared (FTIR) spectrophotometer (Nicolet Is5, Thermo Fisher Scientific, Waltham, MA, USA) was utilized to analyze the chemical structure and interaction between pullulan and PVA, as well as between these polymers and rutin, through the reflection mode with a nanofibrous membrane. All spectra were recorded at $1 \mathrm{~cm}^{-1}$ intervals in the scanning range of $4000-600 \mathrm{~cm}^{-1}$. The mechanical properties of the tensile stress and strain were tested by a tensile machine (Model YG061, Laizhou Electronic Equipment Company, Laizhou, China) at an ambient temperature of $22{ }^{\circ} \mathrm{C}$ and a relative humidity of $65 \%$. The dimensions of the tested samples were $50 \mathrm{~mm}$ in length and $10 \mathrm{~mm}$ in width, while the gauge length between the two holders was $30 \mathrm{~mm}$. The specimen thicknesses were measured using a micrometer having a precision of $0.01 \mathrm{~mm}$. UV transmittance and UV protection factor (UPF) of the electrospun rutin contained nanofibrous mats that were tested with a Textile-UV-performance tester (YG(B)912E, Darong Textile Instrument, Wenzhou, China). The scanned wavenumber ranged from 280 to $400 \mathrm{~nm}( \pm 0.5 \mathrm{~nm})$. The UVA, UVB transmittance, and UPF were recorded automatically after scanning. 


\section{Conclusions}

Pullulan/PVA nanofibrous mats with and without rutin were fabricated via the electrospinning technique. The effect of total concentration, the weight ratio of pullulan/PVA, as well as the different addition amounts of rutin on the formation and diameters of nanofibers were studied. SEM results showed that the average diameter increased as the content of PVA increased. Moreover, the addition and the amount of rutin had no obvious influence on the average diameters when the content was less than $7.41 \%(w / w)$, but formed beaded fibers when the amount was more than $8.54 \%(w / w)$. FTIR analysis confirmed the hydrogen formation between pullulan and PVA molecules, as well as rutin and the pullulan/PVA blend. The mechanical properties showed that the tensile stress increased as strain decreased and the weight ratio of PVA increased. The addition of rutin could enhance the mechanical properties to some extent due to its stiff structure. From a UV-resistant properties test, the incorporation of rutin was able to decrease the transmittance of UVA and UVB to be less than $5 \%$; meanwhile, the value of UPF was above 40 and above 50 when the contents of rutin were $4.46 \%$ and $5.67 \%$, respectively. Therefore, the electrospun pullulan/PVA/rutin nanofibrous mats showed excellent UV resistance and can be used as anti-ultraviolet packaging and outdoor materials.

Acknowledgments: This research was supported by Chinese Scholarship Council (CSC) (Project No. 20145050).

Author Contributions: F.Y. and Y.Q. designed the experiments; Y.Q. and M.Q. performed the experiments and analyzed the SEM images; L.Z. analyzed the data of tensile properties; M.W.K. analyzed the data of FTIR results; L.L. analyzed the data of UV resistant properties; Y.Q. and F.Y. wrote the paper.

Conflicts of Interest: The authors declare no conflict of interest.

\section{References}

1. Park, K.E.; Jung, S.Y.; Lee, S.J.; Min, B.; Park, W.H. Biomimetic nanofibrous scaffolds: Preparation and characterization of chitin/silk fibroin blend nanofibers. Int. J. Biol. Macromol. 2006, 38, 165-173. [CrossRef] [PubMed]

2. Jiang, S.; Liu, F.; Lerch, A.; Ionov, L.; Agarwal, S. Unusual and superfast temperature-triggered actuators. Adv. Mater. 2015, 27, 4865-4870. [CrossRef] [PubMed]

3. Schiffman, J.D.; Schauer, C.L. A review: Electrospinning of biopolymer nanofibers and their applications. Polym. Rev. 2008, 48, 317-352. [CrossRef]

4. Greiner, A.; Wendorff, J.H. Electrospinning: A fascinating method for the preparation of ultrathin fibres. Angew. Chem. Int. Ed. 2007, 46, 5670-5703. [CrossRef] [PubMed]

5. Kachhawa, D.K.; Bhattacharjee, P.; Singhal, R.S. Studies on downstream processing of pullulan. Carbohydr. Polym. 2003, 52, 25-28. [CrossRef]

6. Singh, R.S.; Saini, G.K.; Kennedy, J.F. Pullulan: Microbial sources, production and application. Carbohydr. Polym. 2008, 73, 515-531. [CrossRef] [PubMed]

7. Leathers, T.D. Biotechnological production and applications of pullulan. Appl. Microbiol. Biotechnol. 2003, 62, 468-473. [CrossRef] [PubMed]

8. Islam, M.S.; Yeum, J.H.; Das, A.K. Effect of pullulan/poly(vinyl alcohol) blend system on the montmorillonite structure with property characterization of electrospun pullulan/poly(vinyl alcohol)/montmorillonite nanofibers. J. Colloid Interface Sci. 2012, 368, 273-281. [CrossRef] [PubMed]

9. Karim, M.R.; Lee, H.W.; Kim, R.; Ji, B.C.; Cho, J.W.; Son, T.W.; Oh, W.; Yeu, J.H. Preparation and characterization of electrospun pullulan/montmorillonite nanofiber mats in aqueous solution. Carbohydr. Polym. 2009, 78, 336-342. [CrossRef]

10. Islam, M.S.; Akter, N.; Karim, M.R. Preparation of superhydrophobic membranes by electrospinning of fluorinated silane functionalized pullulan. Colloids Surf. A 2010, 362, 117-120. [CrossRef]

11. Karim, M.R.; Islam, M.S. Thermal Behavior with Mechanical Property of Fluorinated Silane Functionalized Superhydrophobic Pullulan/Poly(vinyl alcohol) Blends by Electrospinning Method. J. Nanomater. 2011, 2011, 979458. [CrossRef] 
12. Lee, H.W.; Karim, M.R.; Ji, H.M.; Choi, J.H.; Ghim, H.D.; Park, S.M.; Oh, W.; Yeum, J.H. Electrospinning fabrication and characterization of Poly(vinyl alcohol)/montmorillonite nanofiber mats. J. Appl. Polym. Sci. 2009, 113, 1860-1867. [CrossRef]

13. Mahmoodi, N.M.; Moktari-Shourijeh, Z. Preparation of PVA-chitosan blend nanofiber and its dye removal ability from colored wastewater. Fibers Polym. 2015, 6, 1861-1869. [CrossRef]

14. Sousa, A.M.M.; Souza, H.K.S.; Uknalis, J.; Liu, S.C.; Goncalves, M.P.; Liu, L.S. Electrospinning of agar/PVA aqueous solutions and its relation with rheological properties. Carbohydr. Polym. 2015, 115, 348-355. [CrossRef] [PubMed]

15. Wang, P.; He, J.H. Electrospun polyvinyl alcohol-milk nanofibers. Therm. Sci. 2013, 17, 1515-1516. [CrossRef]

16. Wang, P.; He, J.H. Electrospun polyvinyl alcohol-honey nanofibers. Therm. Sci. 2013, 17, 1549-1550. [CrossRef]

17. Wang, X.; Yue, T.; Lee, T. Development of Pleurocidin-poly(vinyl alcohol) electrospun antimicrobial nanofibers to retain antimicrobial activity in food system application. Food Control 2015, 54, 150-157. [CrossRef]

18. Arima, H.; Ashida, H.; Danno, G. Rutin-enhanced Antibacterial Activities of Flavonoids against Bacillus cereus and Salmonella enteritidis. Biosci. Biotechnol. Biochem. 2002, 66, 1009-1014. [CrossRef] [PubMed]

19. Roy, S.; Majumdar, S.; Singh, A.K.; Ghosh, B.; Ghosh, N.; Manna, S.; Chakraborty, T.; Mallick, S. Synthesis, characterization, antioxidant status, and toxicity study of vanadium-rutin complex in Balb/c Mice. Biol. Trace Elem. Res. 2015, 166, 183-200. [CrossRef] [PubMed]

20. Kostyuk, V.A.; Potapovich, A.I.; Lulli, D.; Stancato, A.; de Luca, C.; Pastore, S.; Korkina, L. Modulation of human keratinocyte responses to solar UV by plant polyphenol as a basis for chemoprevention of non-melanoma skin cancers. Curr. Med. Chem. 2013, 20, 869-879. [CrossRef] [PubMed]

21. Suzuki, T.; Morishita, T.; Kim, S.J.; Park, S.U.; Woo, S.H.; Noda, T.; Takigawa, S. Physiological roles of rutin in the buckwheat plant. Jpn. Agric. Res. Q. 2015, 49, 37-43. [CrossRef]

22. Xing, Z.C.; Koo, T.H.; Kim, Y.J.; Kwon, O.H.; Kang, I.K. Surface modification of PLGA nanofibrous biocomposites using flavonoids for biomedical applications. J. Adhes. Sci. Technol. 2013, 27, 1382-1392. [CrossRef]

23. Zong, X.; Kim, K.; Fang, D.; Ran, S.; Hsiao, B.S.; Chu, B. Structure and process relationship of electrospun bioabsorbable nanofiber membranes. Polymer 2002, 43, 4403-4412. [CrossRef]

24. Xu, J.; Zhang, J.; Gao, W.; Liang, H.; Wang, H.; Li, J. Preparation of chitosan/PVA blend micro/nanofibers by electrospinning. Mater. Lett. 2009, 63, 658-660. [CrossRef]

25. Zhang, K.; Wang, H.; Huang, C.; Su, Y.; Mo, X.; Ikada, Y. Fabrication of silk fibroin blended P(LLA-CL) nanofibrous scaffolds for tissue engineering. J. Biomed. Mater. Res. A 2010, 93, 984-993. [CrossRef] [PubMed]

26. Calabro, M.L.; Tommasini, S.; Donato, P.; Stancanelli, R.; Raneri, D.; Catania, S.; Costa, C.; Villari, V.; Ficarra, P.; Ficarra, R. The rutin/ $\beta$-cyclodextrin interactions in fully aqueous solution: Spectroscopic studies and biological assays. J. Pharm. Biomed. Anal. 2005, 36, 1019-1027. [CrossRef] [PubMed]

27. Liu, X.; Song, R.; Yu, W.; Pan, N. Developing UV-protective cotton fabric based on SiOx nanoparticles. Fibers Polym. 2012, 13, 489-494. [CrossRef]

(C) 2016 by the authors; licensee MDPI, Basel, Switzerland. This article is an open access article distributed under the terms and conditions of the Creative Commons Attribution (CC-BY) license (http://creativecommons.org/licenses/by/4.0/). 